\title{
A qualitative study on generalized Caputo fractional integro-differential equations
}

\author{
Mohammed D. Kassim ${ }^{1}$, Thabet Abdeljawad ${ }^{2,3,4^{*}}$ (D), Wasfi Shatanawi ${ }^{2}$, Saeed M. Ali ${ }^{1}$ and \\ Mohammed S. Abdo ${ }^{5}$
}

\author{
"Correspondence: \\ tabdeljawad@psu.edu.sa \\ ${ }^{2}$ Department of Mathematics and \\ General Sciences, Prince Sultan \\ University, Riyadh, Saudi Arabia \\ ${ }^{3}$ Department of Medical Research, \\ China Medical University, Taichung \\ 40402, Taiwan \\ Full list of author information is \\ available at the end of the article
}

\begin{abstract}
The aim of this article is to discuss the uniqueness and Ulam-Hyers stability of solutions for a nonlinear fractional integro-differential equation involving a generalized Caputo fractional operator. The used fractional operator is generated by iterating a local integral of the form $\left(l_{a}^{\rho} f\right)(t)=\int_{a}^{t} f(s) s^{\rho-1} d s$. Our reported results are obtained in the Banach space of absolutely continuous functions that rely on Babenko's technique and Banach's fixed point theorem. Besides, our main findings are illustrated by some examples.
\end{abstract}

MSC: 34E10; 34A08; 26A33; 34A12

Keywords: Fractional differential equation; Generalized fractional derivative; Fixed point approach; Babenko's technique

\section{Introduction}

Fractional calculus has gotten much consideration from analysts and engineers, as well as it provided important tools for various areas of applied mathematics, physics, and engineering. Fractional differential equations (FDEs) are used to study plentiful phenomena such as fluid mechanics, plasma physics, optical fibers, biology, flow in nonlinear electric circuits, nonlinear oscillations of the earthquake, mechanics, aerodynamics, regular variations in thermodynamics, etc. Actually, the transform from theoretical to the application aspect of fractional calculus was strongly apparent in the works of Bagley and Torvik in [1-3]. In this regard, the researchers studied many models and used fractional-order derivatives to describe the solution of them. For instance, studying the qualitative properties of solutions of various kinds of FDEs.

Various problems may be modeled by fractional integro-differential equations (FIDEs) such as those representing applications in science and engineering. Up to a recent time, numerous analysts and researchers have discussed the FDEs and FIDEs and got many interesting outcomes utilizing a wide range of fixed point techniques, for instance, Zhang et al. [4], Ahmad et al. [5], Benchohra et al. [6, 7], Ravichandran et al. [8], Trujillo et al. [9], and the following recent papers series [10-18].

Some similar techniques have been applied to get interesting results about some different types of FDEs, see [19-23]. The existence and stability of solutions were studied

(c) The Author(s) 2021. This article is licensed under a Creative Commons Attribution 4.0 International License, which permits use sharing, adaptation, distribution and reproduction in any medium or format, as long as you give appropriate credit to the original author(s) and the source, provide a link to the Creative Commons licence, and indicate if changes were made. The images or other third party material in this article are included in the article's Creative Commons licence, unless indicated otherwise in a credit line to the material. If material is not included in the article's Creative Commons licence and your intended use is not permitted by statutory regulation or exceeds the permitted use, you will need to obtain permission directly from the copyright holder. To view a copy of this licence, visit http://creativecommons.org/licenses/by/4.0/. 
for many of FDEs with some generalized fractional operators in [24-32]. Also here we refer to some recent works that have dealt with Hadamard fractional derivative [33-39]. For instance, Li [38] investigated the uniqueness of solutions of integral equations with Hadamard-type, that is,

$$
a_{n} I_{a}^{\alpha_{n}} \varpi(\varkappa)+\cdots+a_{1} I_{a}^{\alpha_{1}} \varpi(\varkappa)+\varpi(\varkappa)=\mathbb{G}(\varkappa, \varpi(\varkappa)) .
$$

It is realized that the standard definitions for fractional derivatives (Caputo, RiemannLiouville, etc.), which are introduced in the classical monographs, do not fulfill the index law. A few analysts proposed that a differential operator cannot be known as a derivative or fractional derivative if it does not fulfill the index law, see [40]. However, there are some special cases that have been studied on smooth function spaces that make these operators subject to some laws. In this regard, the considered fractional (so-called Katugampola [41, 42]) operator generalizes both the Riemann-Liouville and Hadamard fractional operators in one form, and it is also most regarding the Erdélyi-Kober fractional operator, especially, when $\rho=1$, we get a Caputo fractional derivative, and doing $\rho \downarrow 0$, we get a CaputoHadamard fractional derivative. Consequently, the current results are a generalization of the works of $\mathrm{Li}[38,39]$ and inspired by $[43,44]$. Motivated by the aforesaid discussion, in this research paper, we concentrate on the uniqueness and Ulam-Hyers stability results for the nonlinear FIDEs of the form

$$
\left\{\begin{array}{l}
{ }^{C} D_{a}^{\rho, \alpha_{n}} \varpi(\varkappa)+a_{n-1}{ }^{C} D_{a}^{\rho, \alpha_{n-1}} \varpi(\varkappa)+\cdots+a_{0}{ }^{C} D_{a}^{\rho, \alpha_{0}} \varpi(\varkappa) \\
\quad=\int_{a}^{\varkappa} \mathbb{F}\left(\tau, \varpi^{\prime}(\tau)\right) d \tau, \\
\varpi(a)=0,
\end{array}\right.
$$

where $0<\alpha_{i}<1, i=0,1, \ldots, n, n \in \mathbb{N},{ }^{C} D_{a}^{\rho, \sigma}$ is the generalized Caputo fractional derivative of order $\sigma(>0) \in\left\{\alpha_{i} ; i=0,1, \ldots, n\right\}$ generated by local integrals of the form $\left(I_{a}^{\rho} f\right)(t)=$ $\int_{a}^{t} f(s) s^{\rho-1} d s$, and $\mathbb{F}:[a, b] \times \mathbb{R} \rightarrow \mathbb{R}$ is a continuous function.

There is an absence of various analytical strategies to obtain the qualitative properties of solutions to such problems under generalized fractional operators. To fill this vacuum, we are keen to obtain the existence of a unique solution and the Ulam-Hyers stability of a solution to (1.1). Compared with preceding investigations of such problems, (1.1) is more general because it has a generalized fractional operators. Moreover, the current results are obtained in the Banach space of absolutely continuous functions along with Banach's fixed point technique and Babenko's method [45].

The article is organized as follows: In Sect. 2 we present some necessary tools about the essential properties of generalized fractional operators and the abstract function spaces, in which we aim to employ our analysis techniques. Sections 3 and 4 are devoted to our main analysis results and their illustrated examples. Finally, Sect. 5 contains our short conclusion.

\section{Preliminaries}

In this section, we briefly recall some definitions, lemmas, properties, notations, and wellknown estimations that we will use later.

Let $-\infty<a<b<\infty$. Let $A C[a, b]$ denote the space of absolutely continuous functions on $[a, b][46]$. We denote by $L^{p}(a, b), p \geq 1$, the spaces of Lebesgue integrable functions on $(a, b)$ [46]. 
Definition 2.1 ([46, $(1,9,27),(1,9,28)])$ The so-called multivariate Mittag-Leffler function $E_{\left(a_{1}, \ldots, a_{n}\right), b}\left(z_{1}, \ldots, z_{n}\right)$ of $n$ complex variables $z_{1}, \ldots, z_{n} \in \mathbb{C}$ with complex parameters $a_{1}, \ldots, a_{n}, b \in \mathbb{C}$ is defined by

$$
E_{\left(a_{1}, \ldots, a_{n}\right), b}\left(z_{1}, \ldots, z_{n}\right)=\sum_{\kappa=0}^{\infty} \sum_{\kappa_{1}+\cdots+\kappa_{n}=\kappa}\left(\begin{array}{c}
\kappa \\
\kappa_{1}, \kappa_{2}, \ldots, \kappa_{k}
\end{array}\right) \frac{\prod_{j=1}^{n} z_{j}^{\kappa_{j}}}{\Gamma\left(b+\sum_{j=1}^{n} a_{j} \kappa_{j}\right)},
$$

in terms of multinomial coefficients

$$
\left(\begin{array}{c}
\kappa \\
\kappa_{1}, \kappa_{2}, \ldots, \kappa_{k}
\end{array}\right)=\frac{\kappa !}{\kappa_{1} ! \kappa_{2} ! \ldots \kappa_{k} !}, \quad \kappa, \kappa_{1}, \ldots, \kappa_{k} \in \mathbb{N}_{0} .
$$

Theorem 2.2 ([47] (Multinomial theorem)) For a positive integer $n$ and a nonnegative integer $k$,

$$
\left(z_{1}+z_{2}+\cdots+z_{k}\right)^{n}=\sum_{\kappa_{1}+\kappa_{2}+\cdots+\kappa_{k}=n}\left(\begin{array}{c}
n \\
\kappa_{1}, \kappa_{2}, \ldots, \kappa_{k}
\end{array}\right) \prod_{j=1}^{k} z_{j}^{\kappa_{j}} .
$$

Definition 2.3 ([48, $(6,3,1)]$ (Babenko's method)) Given the FDE

$$
\left(1+\lambda I_{0}^{\alpha}\right) \vartheta(\varkappa)=f(\varkappa)
$$

where $\alpha>0, \lambda$ is a constant, and $I_{0}^{\alpha} \vartheta(\varkappa)=\frac{1}{\Gamma(\alpha)} \int_{0}^{\varkappa}(\varkappa-\tau)^{\alpha-1} \vartheta(\tau) d \tau$. The solution of (2.2) is

$$
\vartheta(\varkappa)=\left(1+\lambda I^{\alpha}\right)^{-1} f(\varkappa)
$$

where $\left(1+\lambda I^{\alpha}\right)^{-1}$ denotes the left inverse operator to the operation $\left(1+\lambda D^{-\alpha}\right)$. Using the binomial expansion of $\left(1+\lambda I^{\alpha}\right)^{-1}$, solution (2.3) can be expressed by

$$
\vartheta(\varkappa)=\sum_{n=0}^{\infty}(-1)^{n} \lambda^{n} I^{\alpha n} f(\varkappa)
$$

Definition 2.4 ([49] (Dirichlet formula)) Let $f$ be a continuous function on $[a, b]$ and $\alpha, \beta>0$. Then

$$
\begin{aligned}
& \int_{a}^{\varkappa}(\varkappa-\tau)^{\alpha-1} d \tau \int_{a}^{\tau}(\tau-s)^{\beta-1} f(\tau, s) d s \\
& =\int_{a}^{\varkappa} d s \int_{s}^{\varkappa}(\varkappa-\tau)^{\alpha-1}(\tau-s)^{\beta-1} f(\tau, s) d \tau .
\end{aligned}
$$

Define the Banach space (see $[46,(1,9,27),(1,9,28)])$

$$
A C_{0}[a, b]=\left\{\varpi: \varpi(\tau) \in A C[a, b] \text { with } \varpi(a)=0 \text { and }\|\varpi\|_{0}=\int_{a}^{b}\left|\varpi^{\prime}(\tau)\right| d \tau<\infty\right\} .
$$

Next, we introduce some definitions, notation, and properties of the generalized fractional integral and derivative. 
Definition 2.5 ([41]) The generalized fractional integral and derivative are defined, respectively, by

$$
{ }^{\rho} I_{a}^{\alpha} \vartheta(\varkappa)=\frac{\rho^{1-\alpha}}{\Gamma(\alpha)} \int_{a}^{\varkappa}\left(\varkappa^{\rho}-\tau^{\rho}\right)^{\alpha-1} \tau^{\rho-1} \vartheta(\tau) d \tau, \quad \alpha>0, \rho>0,
$$

and

$$
\begin{aligned}
{ }^{C} D_{a}^{\rho, \alpha} \vartheta(\varkappa) & =\left({ }^{\rho} I_{a}^{n-\alpha} \delta_{\rho}^{n} \vartheta\right)(\varkappa) \\
& =\frac{\rho^{1-n+\alpha}}{\Gamma(n-\alpha)} \int_{a}^{\varkappa}\left(\varkappa^{\rho}-\tau^{\rho}\right)^{n-\alpha-1} \tau^{\rho-1} \delta_{\rho}^{n} \vartheta(\tau) d \tau, \quad \alpha>0, \rho>0,
\end{aligned}
$$

where

$$
n=-[-\alpha], \quad \delta_{\rho}^{n}=\left(\varkappa^{1-\rho} \frac{d}{d \varkappa}\right)^{n} .
$$

Definition 2.6 ([50]) The incomplete gamma function is defined by

$$
\gamma(\alpha, \tau)=\int_{0}^{\tau} s^{\alpha-1} e^{-s} d s=\tau^{\alpha} \Gamma(\alpha) e^{-\tau} \sum_{i=0}^{\infty} \frac{\tau^{i}}{\Gamma(\alpha+i+1)}, \quad \alpha>0, \tau \geq 0 .
$$

Property 2.7 ([44]) If $\alpha \geq 0, \rho>0$, and $\beta>0$, then

$$
\begin{aligned}
& { }^{\rho} I_{a}^{\alpha}\left(\frac{\varkappa^{\rho}-a^{\rho}}{\rho}\right)^{\beta}=\frac{\Gamma(\beta+1)}{\Gamma(\beta+\alpha+1)}\left(\frac{\varkappa^{\rho}-a^{\rho}}{\rho}\right)^{\beta+\alpha}, \quad \varkappa>a, \\
& { }^{C} D_{a}^{\rho, \alpha}\left(\frac{\varkappa^{\rho}-a^{\rho}}{\rho}\right)^{\beta}=\frac{\Gamma(\beta+1)}{\Gamma(\beta-\alpha+1)}\left(\frac{\varkappa^{\rho}-a^{\rho}}{\rho}\right)^{\beta-\alpha}, \quad \varkappa>a .
\end{aligned}
$$

The generalized fractional operators in Definition 2.5 fulfill the following properties.

Property 2.8 ([51]) Let $\alpha, \rho$, and $\beta>0$. If $\vartheta \in A C_{0}[a, b]$, then

$$
\begin{aligned}
& { }^{C} D_{a}^{\rho, \alpha \rho} I_{a}^{\alpha} \vartheta(\tau)=\vartheta(\tau), \quad \tau>a, \\
& { }^{\rho} I_{a}^{\alpha \rho} I_{a}^{\beta} \vartheta(\tau)={ }^{\rho} I_{a}^{\alpha+\beta} \vartheta(\tau), \quad \tau>a, \\
& { }^{C} D_{a}^{\rho, \beta \rho} I_{a}^{\alpha} \vartheta(\tau)={ }^{\rho} I_{a}^{\alpha-\beta} \vartheta(\tau), \quad \alpha>\beta, \tau>a,
\end{aligned}
$$

and

$$
{ }^{\rho} I_{a}^{\alpha} \vartheta(a)=0
$$

Lemma 2.9 Let $\alpha, \beta \in[0,1]$ and $\rho>0$. If $\vartheta \in A C_{0}[a, b]$, then

$$
\begin{aligned}
& { }^{\rho} I_{a}^{\alpha C} D_{a}^{\rho, \alpha} \vartheta(\tau)=\vartheta(\tau), \quad \tau>a, \\
& { }^{\rho} I_{a}^{\alpha C} D_{a}^{\rho, \beta} \vartheta(\tau)={ }^{\rho} I_{a}^{\alpha-\beta} \vartheta(\tau), \quad \alpha>\beta, \tau>a .
\end{aligned}
$$


Proof Let $\vartheta \in A C_{0}[a, b]$. From Definition 2.5 and Property 2.8, we have

$$
{ }^{\rho} I_{a}^{\alpha C} D_{a}^{\rho, \alpha} \vartheta(\tau)={ }^{\rho} I_{a}^{\alpha \rho} I_{a}^{1-\alpha} \delta_{\rho}^{1} \vartheta(\tau)={ }^{\rho} I_{a}^{1} \delta_{\rho}^{1} \vartheta(\tau)=\vartheta(\tau)-\vartheta(a)=\vartheta(\tau), \quad \tau>a,
$$

and

$$
\begin{aligned}
{ }^{\rho} I_{a}^{\alpha C} D_{a}^{\rho, \beta} \vartheta(\tau) & ={ }^{\rho} I_{a}^{\alpha \rho} I_{a}^{1-\beta} \delta_{\rho}^{1} \vartheta(\tau)={ }^{\rho} I_{a}^{\alpha-\beta \rho} I_{a}^{1} \delta_{\rho}^{1} \vartheta(\tau) \\
& ={ }^{\rho} I_{a}^{\alpha-\beta}(\vartheta(\tau)-\vartheta(a))={ }^{\rho} I_{a}^{\alpha-\beta} \vartheta(\tau), \quad \alpha>\beta, \tau>a .
\end{aligned}
$$

Lemma 2.10 Let $\alpha, \rho>0$. Then ${ }^{\rho} I_{a}^{\alpha}$ is bounded from $A C_{0}[a, b]$ into $A C_{0}[a, b]$ and

$$
\left\|{ }^{\rho} I_{a}^{\alpha} \vartheta\right\|_{0} \leq \frac{1}{\Gamma(\alpha+1)}\left(\frac{b^{\rho}-a^{\rho}}{\rho}\right)^{\alpha}\|\vartheta\|_{0}
$$

Proof Let $\vartheta \in A C_{0}[a, b]$. Then

$$
\vartheta(\tau)=\int_{a}^{\tau} \vartheta^{\prime}(s) d s=\int_{a}^{\tau} \theta(s) d s, \quad \theta(\tau)=\vartheta^{\prime}(\tau), \quad \text { and } \quad \vartheta(a)=0 \text {. }
$$

From Definition 2.5, we obtain

$$
\begin{aligned}
{ }^{\rho} I_{a}^{\alpha} \vartheta(\varkappa) & ={ }^{\rho} I_{a}^{\alpha}\left(\int_{a}^{\tau} \theta(s) d s\right)(\varkappa) \\
& =\frac{\rho^{1-\alpha}}{\Gamma(\alpha)} \int_{a}^{\varkappa}\left(\varkappa^{\rho}-\tau^{\rho}\right)^{\alpha-1} \tau^{\rho-1} \int_{a}^{\tau} \theta(s) d s d \tau .
\end{aligned}
$$

Using Dirichlet's formula (2.5), we obtain

$$
\begin{aligned}
{ }^{\rho} I_{a}^{\alpha} \vartheta(\varkappa) & =\frac{\rho^{1-\alpha}}{\Gamma(\alpha)} \int_{a}^{\varkappa} \theta(s) \int_{s}^{\varkappa}\left(\varkappa^{\rho}-\tau^{\rho}\right)^{\alpha-1} \tau^{\rho-1} d \tau d s \\
& =\frac{\rho^{1-\alpha}}{\Gamma(\alpha)} \int_{a}^{\varkappa} \theta(s)\left[-\frac{\left(\varkappa^{\rho}-\tau^{\rho}\right)^{\alpha}}{\alpha \rho}\right]_{\tau=s}^{\varkappa} d s \\
& =\frac{\rho^{1-\alpha}}{\Gamma(\alpha)} \int_{a}^{\varkappa} \theta(s)\left[\frac{\left(\varkappa^{\rho}-s^{\rho}\right)^{\alpha}}{\alpha \rho}\right] d s \\
& \leq \frac{1}{\Gamma(\alpha+1)}\left(\frac{b^{\rho}-a^{\rho}}{\rho}\right)^{\alpha} \int_{a}^{\varkappa}|\theta(s)| d s \\
& =\frac{1}{\Gamma(\alpha+1)}\left(\frac{b^{\rho}-a^{\rho}}{\rho}\right)^{\alpha} \int_{a}^{\varkappa}\left|\vartheta^{\prime}(s)\right| d s \\
& =\frac{1}{\Gamma(\alpha+1)}\left(\frac{b^{\rho}-a^{\rho}}{\rho}\right)^{\alpha}\|\vartheta\|_{0} .
\end{aligned}
$$

Next, we prove the following useful lemma.

Lemma 2.11 If $\alpha \geq 0, \rho>0$, then

$$
{ }^{\rho} I_{a}^{\alpha} e^{\varkappa^{\rho}}=e^{a^{\rho}}\left(\frac{\varkappa^{\rho}-a^{\rho}}{\rho}\right)^{\alpha} \sum_{i=0}^{\infty} \frac{\left(\varkappa^{\rho}-a^{\rho}\right)^{i}}{\Gamma(\alpha+i+1)} .
$$


Proof By Definition 2.5, we have

$$
{ }^{\rho} I_{a}^{\alpha} e^{\varkappa^{\rho}}=\frac{1}{\Gamma(\alpha)} \int_{a}^{\varkappa}\left(\frac{\varkappa^{\rho}-\tau^{\rho}}{\rho}\right)^{\alpha-1} \tau^{\rho-1} e^{\tau^{\rho}} d \tau .
$$

Making the change of the variable

$$
s=\frac{\varkappa^{\rho}-\tau^{\rho}}{\rho} \Rightarrow \tau^{\rho}=\varkappa^{\rho}-\rho s .
$$

Therefore,

$$
{ }^{\rho} I_{a}^{\alpha} e^{\varkappa^{\rho}}=\frac{1}{\Gamma(\alpha)} \int_{0}^{\left(\varkappa^{\rho}-a^{\rho}\right) / \rho} s^{\alpha-1} e^{\varkappa^{\rho}-\rho s} d s=\frac{e^{\varkappa^{\rho}}}{\Gamma(\alpha)} \int_{0}^{\left(\varkappa^{\rho}-a^{\rho}\right) / \rho} s^{\alpha-1} e^{-\rho s} d s
$$

Let $r=\rho s$. Then

$$
{ }^{\rho} I_{a}^{\alpha} e^{\varkappa^{\rho}}=\frac{e^{\varkappa^{\rho}}}{\rho^{\alpha} \Gamma(\alpha)} \int_{0}^{\left(\varkappa^{\rho}-a^{\rho}\right)} r^{\alpha-1} e^{-r} d r
$$

Using Definition 2.6, we get

$$
{ }^{\rho} I_{a}^{\alpha} e^{\varkappa^{\rho}}=\gamma\left(\alpha, \varkappa^{\rho}-a^{\rho}\right) \frac{e^{\varkappa^{\rho}}}{\rho^{\alpha} \Gamma(\alpha)}=e^{a^{\rho}}\left(\frac{\varkappa^{\rho}-a^{\rho}}{\rho}\right)^{\alpha} \sum_{i=0}^{\infty} \frac{\left(\varkappa^{\rho}-a^{\rho}\right)^{i}}{\Gamma(\alpha+i+1)} .
$$

\section{Main results}

Theorem 3.1 Suppose $a_{i} \in \mathbb{C}(i=0,1, \ldots, n-1)$ with $0<\alpha_{0}<\alpha_{1}<\cdots<\alpha_{n}<1$. If $g \in$ $A C_{0}[a, b]$, then the linear problem

$$
\left\{\begin{array}{l}
{ }^{C} D_{a}^{\rho, \alpha_{n}} \varpi(\varkappa)+a_{n-1}{ }^{C} D_{a}^{\rho, \alpha_{n-1}} \varpi(\varkappa)+\cdots+a_{0}{ }^{C} D_{a}^{\rho, \alpha_{0}} \varpi(\varkappa)=g(\varkappa), \\
\varpi(a)=0
\end{array}\right.
$$

has a solution

$$
\begin{aligned}
\varpi(\varkappa)= & \sum_{J=0}^{\infty}(-1)^{J} \sum_{J_{1}+\cdots+J_{n}=J}\left(\begin{array}{c}
J \\
J_{1}, J_{2}, \ldots, J_{n}
\end{array}\right) \\
& \times a_{n-1}^{J_{1}} \cdots a_{0}^{J_{n} \rho} I_{a}^{J_{1}\left(\alpha_{n}-\alpha_{n-1}\right)+\cdots+J_{n}\left(\alpha_{n}-\alpha_{0}\right)+\alpha_{n}} g(\varkappa) .
\end{aligned}
$$

Proof Applying ${ }^{\rho} I_{a}^{\alpha_{n}}$ to both sides of (3.1), we find that

$$
{ }^{\rho} I_{a}^{\alpha_{n} C} D_{a}^{\rho, \alpha_{n}} \varpi(\varkappa)+a_{n-1}{ }^{\rho} I_{a}^{\alpha_{n} C} D_{a}^{\rho, \alpha_{n-1}} \varpi(\varkappa)+\cdots+a_{0}{ }^{\rho} I_{a}^{\alpha_{n} C} D_{a}^{\rho, \alpha_{0}} \varpi(\varkappa)={ }^{\rho} I_{a}^{\alpha_{n}} g(\varkappa) .
$$

Using Lemma 2.9, we obtain

$$
\varpi(\varkappa)+a_{n-1}{ }^{\rho} I_{a}^{\alpha_{n}-\alpha_{n-1}} \varpi(\varkappa)+\cdots+a_{0}{ }^{\rho} I_{a}^{\alpha_{n}-\alpha_{0}} \varpi(\varkappa)={ }^{\rho} I_{a}^{\alpha_{n}} g(\varkappa) .
$$

By noting that $\varpi(a)=0$ and $0<\alpha_{0}<\alpha_{1}<\cdots<\alpha_{n}<1$, then

$$
\left(1+a_{n-1}{ }^{\rho} I_{a}^{\alpha_{n}-\alpha_{n-1}}+\cdots+a_{0}{ }^{\rho} I_{a}^{\alpha_{n}-\alpha_{0}}\right) \varpi(\varkappa)={ }^{\rho} I_{a}^{\alpha_{n}} g(\varkappa) .
$$


Thanks to Babenko's method, we have

$$
\varpi(\varkappa)=\left(1+a_{n-1}^{\rho} I_{a}^{\alpha_{n}-\alpha_{n-1}}+\cdots+a_{0}^{\rho} I_{a}^{\alpha_{n}-\alpha_{0}}\right)^{-1} \rho I_{a}^{\alpha_{n}} g(\varkappa) .
$$

Multinomial theorem and Property 2.8 give

$$
\begin{aligned}
& \varpi(\varkappa)=\sum_{J=0}^{\infty}(-1)^{\jmath}\left(a_{n-1}{ }^{\rho} I_{a}^{\alpha_{n}-\alpha_{n-1}}+\cdots+a_{0}{ }^{\rho} I_{a}^{\alpha_{n}-\alpha_{0}}\right)^{\jmath} \rho I_{a}^{\alpha_{n}} g(\varkappa) \\
& =\sum_{j=0}^{\infty}(-1)^{J} \sum_{J_{1}+\cdots+J_{n}=J}\left(\begin{array}{c}
J \\
J_{1}, J_{2}, \ldots, J_{n}
\end{array}\right)
\end{aligned}
$$

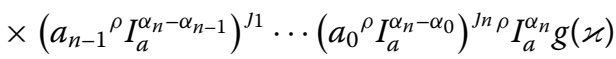

$$
\begin{aligned}
& =\sum_{J=0}^{\infty}(-1)^{J} \sum_{J_{1}+\cdots+J n=J}\left(\begin{array}{c}
J \\
J_{1}, J_{2}, \ldots, J_{n}
\end{array}\right)
\end{aligned}
$$

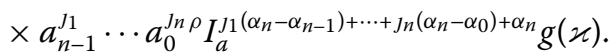

By taking the limit as $\varkappa \rightarrow a$, we obtain $\varpi(a)=0$. It remains to show that the series converges in the space $A C_{0}[a, b]$ and is absolutely continuous on $[a, b]$. By Lemma 2.10

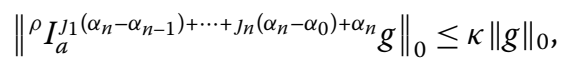

where

$$
\kappa=\frac{\left(\frac{b^{\rho}-a^{\rho}}{\rho}\right)^{J_{1}\left(\alpha_{n}-\alpha_{n-1}\right)+\cdots+J_{n}\left(\alpha_{n}-\alpha_{0}\right)+\alpha_{n}}}{\Gamma\left(J_{1}\left(\alpha_{n}-\alpha_{n-1}\right)+\cdots+J_{n}\left(\alpha_{n}-\alpha_{0}\right)+\alpha_{n}+1\right)} .
$$

Then

$$
\begin{aligned}
\|\varpi\|_{0} \leq & \kappa \sum_{j=0}^{\infty} \sum_{J_{1}+\cdots+J_{n}=J}\left(\begin{array}{c}
J \\
J_{1}, J_{2}, \ldots, J_{n}
\end{array}\right)\left|a_{n-1}^{J_{1}}\right| \cdots\left|a_{0}^{J_{n}}\right| \\
& \times \frac{\left(\frac{b^{\rho}-a^{\rho}}{\rho}\right)^{J_{1}\left(\alpha_{n}-\alpha_{n-1}\right)+\cdots+J_{n}\left(\alpha_{n}-\alpha_{0}\right)+\alpha_{n}}}{\Gamma\left(J_{1}\left(\alpha_{n}-\alpha_{n-1}\right)+\cdots+J_{n}\left(\alpha_{n}-\alpha_{0}\right)+\alpha_{n}+1\right)}\|g\|_{0} \\
= & \kappa \sum_{J=0}^{\infty} \sum_{J_{1}+\cdots+J_{n}=J}\left(\begin{array}{c}
J \\
J_{1}, J_{2}, \ldots, J_{n}
\end{array}\right) \\
& \times \frac{\left(\left|a_{n-1}\right|\left(\frac{b^{\rho}-a^{\rho}}{\rho}\right)^{\left(\alpha_{n}-\alpha_{n-1}\right)}\right)^{J_{1}} \ldots\left(\left|a_{0}\right|\left(\frac{b^{\rho}-a^{\rho}}{\rho}\right)^{\left(\alpha_{n}-\alpha_{0}\right)}\right)^{J_{n}}}{\Gamma\left(J_{1}\left(\alpha_{n}-\alpha_{n-1}\right)+\cdots+J_{n}\left(\alpha_{n}-\alpha_{0}\right)+\alpha_{n}+1\right)}\|g\|_{0} \\
= & \kappa E_{\left(\alpha_{n}-\alpha_{n-1}, \ldots, \alpha_{n}-\alpha_{0}, \alpha_{n}+1\right)}\left(\left|a_{n-1}\right|\left(\frac{b^{\rho}-a^{\rho}}{\rho}\right)^{\alpha_{n}-\alpha_{n-1}}\right. \\
& \left.\ldots,\left|a_{0}\right|\left(\frac{b^{\rho}-a^{\rho}}{\rho}\right)^{\alpha_{n}-\alpha_{0}}\right)\|g\|_{0},
\end{aligned}
$$


where

$$
E_{\left(\alpha_{n}-\alpha_{n-1}, \ldots, \alpha_{n}-\alpha_{0}, \alpha_{n}+1\right)}\left(\left|a_{n-1}\right|\left(\frac{b^{\rho}-a^{\rho}}{\rho}\right)^{\alpha_{n}-\alpha_{n-1}}, \ldots,\left|a_{0}\right|\left(\frac{b^{\rho}-a^{\rho}}{\rho}\right)^{\alpha_{n}-\alpha_{0}}\right)<\infty
$$

is the value at

$$
v_{1}=\left|a_{n-1}\right|\left(\frac{b^{\rho}-a^{\rho}}{\rho}\right)^{\alpha_{n}-\alpha_{n-1}}, \ldots, v_{n}=\left|a_{0}\right|\left(\frac{b^{\rho}-a^{\rho}}{\rho}\right)^{\alpha_{n}-\alpha_{0}}
$$

of the multivariate Mittag-Leffler function $E_{\left(\alpha_{n}-\alpha_{n-1}, \ldots, \alpha_{n}-\alpha_{0}, \alpha_{n}+1\right)}\left(v_{1}, \ldots, v_{n}\right)$ defined by Eq. (2.1). Thus, the series to the right of Eq. (3.2) is convergent. Clearly, $\varpi(\varkappa) \in A C[a, b]$ since $g \in A C[a, b]$. To confirm that the acquired series is a solution, we substitute it into the left-hand side of Eq. (3.1) as follows:

$$
\begin{aligned}
& { }^{C} D_{a}^{\rho, \alpha_{n}}\left(\sum_{J=0}^{\infty}(-1)^{J} \sum_{J_{1}+\cdots+J_{n}=J}\left(\begin{array}{c}
J \\
J_{1}, J_{2}, \ldots, J_{n}
\end{array}\right)\right. \\
& \left.\times a_{n-1}^{J_{1}} \cdots a_{0}^{J_{n} \rho} I_{a}^{J_{1}\left(\alpha_{n}-\alpha_{n-1}\right)+\cdots+J_{n}\left(\alpha_{n}-\alpha_{0}\right)+\alpha_{n}} g(\varkappa)\right) \\
& +a_{n-1}{ }^{C} D_{a}^{\rho, \alpha_{n-1}}\left(\sum_{j=0}^{\infty}(-1)^{J} \sum_{J_{1}+\cdots+J_{n}=J}\left(\begin{array}{c}
J \\
J_{1}, J_{2}, \ldots, J_{n}
\end{array}\right)\right. \\
& \left.\times a_{n-1}^{J_{1}} \cdots a_{0}^{J_{n} \rho} I_{a}^{J_{1}\left(\alpha_{n}-\alpha_{n-1}\right)+\cdots+J_{n}\left(\alpha_{n}-\alpha_{0}\right)+\alpha_{n}} g(\varkappa)\right) \\
& +\cdots+a_{0}{ }^{C} D_{a}^{\rho, \alpha_{0}}\left(\sum_{j=0}^{\infty}(-1)^{J} \sum_{J_{1}+\cdots+J_{n}=J}\left(\begin{array}{c}
J \\
J_{1}, J_{2}, \ldots, J_{n}
\end{array}\right)\right. \\
& \left.\times a_{n-1}^{J 1} \cdots a_{0}^{J n \rho} I_{a}^{J_{1}\left(\alpha_{n}-\alpha_{n-1}\right)+\cdots+J_{n}\left(\alpha_{n}-\alpha_{0}\right)+\alpha_{n}} g(\varkappa)\right) \\
& ={ }^{C} D_{a}^{\rho, \alpha_{n}}\left({ }^{\rho} I_{a}^{\alpha_{n}} g(\varkappa)+\sum_{j=1}^{\infty}(-1)^{J} \sum_{J_{1}+\cdots+J_{n}=J}\left(\begin{array}{c}
J \\
J_{1}, J_{2}, \ldots, J_{n}
\end{array}\right)\right.
\end{aligned}
$$

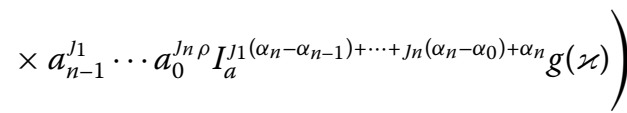

$$
\begin{aligned}
& +\left(\sum_{J=0}^{\infty}(-1)^{J} \sum_{J_{1}+\cdots+J_{n}=J}\left(\begin{array}{c}
J \\
J_{1}, J_{2}, \ldots, J_{n}
\end{array}\right)\right. \\
& \left.\times a_{n-1}^{j 1+1} \cdots a_{0}^{j n \rho} I_{a}^{\left(J_{1}+1\right)\left(\alpha_{n}-\alpha_{n-1}\right)+\cdots+J_{n}\left(\alpha_{n}-\alpha_{0}\right)} g(\varkappa)\right) \\
& +\cdots+\left(\sum_{j=0}^{\infty}(-1)^{J} \sum_{J_{1}+\cdots+J_{n}=J}\left(\begin{array}{c}
J \\
J_{1}, J_{2}, \ldots, J_{n}
\end{array}\right)\right. \\
& \left.\times a_{n-1}^{J_{1}} \cdots a_{0}^{J_{n}+1} \rho_{a}^{J_{1}\left(\alpha_{n}-\alpha_{n-1}\right)+\cdots+\left(J_{n}+1\right)\left(\alpha_{n}-\alpha_{0}\right)} g(\varkappa)\right)
\end{aligned}
$$




$$
\begin{aligned}
& =g(\varkappa)+\sum_{J=1}^{\infty}(-1)^{J} \sum_{J_{1}+\cdots+J_{n}=\jmath}\left(\begin{array}{c}
J \\
J_{1}, J_{2}, \ldots, J_{n}
\end{array}\right) \\
& \quad \times a_{n-1}^{J_{1}} \cdots a_{0}^{J_{n} \rho} I_{a}^{J_{1}\left(\alpha_{n}-\alpha_{n-1}\right)+\cdots+J_{n}\left(\alpha_{n}-\alpha_{0}\right)} g(\varkappa) \\
& \quad+\sum_{J=0}^{\infty}(-1)^{J} \sum_{J_{1}+\cdots+J_{n}=J}\left(\begin{array}{c}
J \\
J_{1}, J_{2}, \ldots, J_{n}
\end{array}\right) \\
& \quad \times a_{n-1}^{J_{1}+1} \cdots a_{0}^{J_{n} \rho} I_{a}^{\left(J_{1}+1\right)\left(\alpha_{n}-\alpha_{n-1}\right)+\cdots+J_{n}\left(\alpha_{n}-\alpha_{0}\right)} g(\varkappa) \\
& +\cdots+\sum_{j=0}^{\infty}(-1)^{J} \sum_{J_{1}+\cdots+J_{n}=\jmath}\left(\begin{array}{c}
J \\
J_{1}, J_{2}, \ldots, J_{n}
\end{array}\right) \\
& \quad \times a_{n-1}^{J_{1}} \cdots a_{0}^{J_{n}+1} I_{a}^{J_{1}\left(\alpha_{n}-\alpha_{n-1}\right)+\cdots+\left(J_{n}+1\right)\left(\alpha_{n}-\alpha_{0}\right)} g(\varkappa) \\
& =g(\varkappa)
\end{aligned}
$$

by the deletion. Observe that all series are absolutely convergent and the term rearrangements are possible for the deletion. In fact,

$$
\begin{aligned}
& -\sum_{J_{1}+\cdots+J_{n}=1}\left(\begin{array}{c}
J \\
J_{1}, J_{2}, \ldots, J_{n}
\end{array}\right) a_{n-1}^{J 1} \cdots a_{0}^{J n \rho} I_{a}^{J 1}\left(\alpha_{n}-\alpha_{n-1}\right)+\cdots+J_{n}\left(\alpha_{n}-\alpha_{0}\right) g(\varkappa) \\
& +\sum_{J_{1}+\cdots+J_{n}=0}\left(\begin{array}{c}
J \\
J_{1}, J_{2}, \ldots, J_{n}
\end{array}\right) a_{n-1}^{J_{1}+1} \cdots a_{0}^{J n \rho} I_{a}^{\left(J_{1}+1\right)\left(\alpha_{n}-\alpha_{n-1}\right)+\cdots+J_{n}\left(\alpha_{n}-\alpha_{0}\right)} g(\varkappa)+\cdots \\
& +\sum_{J_{1}+\cdots+J_{n}=0}\left(\begin{array}{c}
J \\
J_{1}, J_{2}, \ldots, J_{n}
\end{array}\right) a_{n-1}^{J_{1}} \cdots a_{0}^{J_{n}+1} I_{a}^{J_{1}\left(\alpha_{n}-\alpha_{n-1}\right)+\cdots+(J n+1)\left(\alpha_{n}-\alpha_{0}\right)} g(\varkappa) \\
& =0 \text {. }
\end{aligned}
$$

The remnant terms cancel each other comparatively. Obviously, the uniqueness follows directly from the fact that

$$
{ }^{C} D_{a}^{\rho, \alpha_{n}} \varpi(\varkappa)+a_{n-1}{ }^{C} D_{a}^{\rho, \alpha_{n-1}} \varpi(\varkappa)+\cdots+a_{0}{ }^{C} D_{a}^{\rho, \alpha_{0}} \varpi(\varkappa)=0
$$

only has solution zero by Babenko's approach. This finalizes the proof.

Remark 3.2 A solution of Eq. (3.1) in $A C_{0}[a, b]$ is said to be stable if $\forall \epsilon>0 \exists \delta>0$ such that $\|\varpi\|_{0}<\epsilon$ if $\|g\|_{0}<\delta$. Applying the inequality

$$
\begin{gathered}
\|\varpi\|_{0} \leq \kappa E_{\left(\alpha_{n}-\alpha_{n-1}, \ldots, \alpha_{n}-\alpha_{0}, \alpha_{n}+1\right)}\left(\left|a_{n-1}\right|\left(\frac{b^{\rho}-a^{\rho}}{\rho}\right)^{\alpha_{n}-\alpha_{n-1}},\right. \\
\left.\ldots,\left|a_{0}\right|\left(\frac{b^{\rho}-a^{\rho}}{\rho}\right)^{\alpha_{n}-\alpha_{0}}\right)\|g\|_{0},
\end{gathered}
$$

we obtain that $\varpi$ is stable.

Example 3.3 The Katugampola-type FIDE

$$
{ }^{C} D_{a}^{\rho, 0.9} \varpi(\varkappa)+2{ }^{C} D_{a}^{\rho, 0.7} \varpi(\varkappa)-{ }^{C} D_{a}^{\rho, 0.4} \varpi(\varkappa)=\left(\frac{\varkappa^{\rho}-a^{\rho}}{\rho}\right)^{\beta}
$$


has the solution

$$
\begin{aligned}
\varpi(\varkappa)= & \sum_{J=0}^{\infty}(-1)^{J} \sum_{J_{1}+J_{2}=J}\left(\begin{array}{c}
J \\
J_{1}, J_{2}
\end{array}\right)(2)^{J_{1}}(-1)^{J_{2}} \\
& \times \frac{\Gamma(\beta+1)}{\Gamma\left(\beta+0.2 J_{1}+0.5 J_{2}+1.9\right)} \\
& \times\left(\frac{\varkappa^{\rho}-a^{\rho}}{\rho}\right)^{\beta+0.2 J_{1}+0.5 J_{2}+0.9}
\end{aligned}
$$

in $A C_{0}[a, b]$. Indeed, in view of Theorem 3.1, we have

$$
\begin{aligned}
\varpi(\varkappa)= & \sum_{J=0}^{\infty}(-1)^{J} \sum_{J_{1}+J_{2}=\jmath}\left(\begin{array}{c}
J \\
J_{1}, J_{2}
\end{array}\right)(2)^{J 1}(-1)^{J 2} \\
& \times{ }^{\rho} I_{a}^{0.2 J_{1}+0.5 J_{2}+0.9}\left(\frac{\varkappa^{\rho}-a^{\rho}}{\rho}\right)^{\beta} .
\end{aligned}
$$

Using Property 2.7, we obtain

$$
\begin{aligned}
\varpi(\varkappa)= & \sum_{J=0}^{\infty}(-1)^{J} \sum_{J_{1}+J_{2}=J}\left(\begin{array}{c}
J \\
J_{1}, J_{2}
\end{array}\right)(2)^{J_{1}}(-1)^{J_{2}} \\
& \times \frac{\Gamma(\beta+1)}{\Gamma\left(\beta+0.2 J_{1}+0.5 J_{2}+1.9\right)} \\
& \times\left(\frac{\varkappa^{\rho}-a^{\rho}}{\rho}\right)^{\beta+0.2 J_{1}+0.5 J_{2}+0.9}
\end{aligned}
$$

Example 3.4 The Katugampola-type FIDE

$$
{ }^{C} D_{a}^{\rho, 0.8} \varpi(\varkappa)+{ }^{C} D_{a}^{\rho, 0.7} \varpi(\varkappa)-3{ }^{C} D_{a}^{\rho, 0.2} \varpi(\varkappa)=e^{\varkappa^{\rho}}
$$

has the solution

$$
\begin{aligned}
\varpi(\varkappa)= & e^{a^{\rho}} \sum_{j=0}^{\infty}(-1)^{j} \sum_{J_{1}+J_{2}=J}\left(\begin{array}{c}
J \\
J_{1}, J_{2}
\end{array}\right)(-3)^{J 2} \\
& \times\left(\frac{\varkappa^{\rho}-a^{\rho}}{\rho}\right)^{0.1 j_{1}+0.6 J_{2}+0.8} \\
& \times \sum_{i=0}^{\infty} \frac{\left(\varkappa^{\rho}-a^{\rho}\right)^{i}}{\Gamma\left(0.1 J_{1}+0.6 J_{2}+0.8+i+1\right)}
\end{aligned}
$$

in $A C_{0}[a, b]$. Indeed, according to Theorem 3.1, we obtain

$$
\begin{aligned}
\varpi(\varkappa)= & \sum_{j=0}^{\infty}(-1)^{J} \sum_{J 1+J_{2}=\jmath}\left(\begin{array}{c}
J \\
J_{1}, J_{2}
\end{array}\right)(1)^{J_{1}}(-3)^{J 2} \\
& \times{ }^{\rho} I_{a}^{0.1 j_{1}+0.6 J_{2}+0.8} e^{\varkappa^{\rho}} .
\end{aligned}
$$


Using Lemma 2.11, we get

$$
\begin{aligned}
\varpi(\varkappa)= & \sum_{J=0}^{\infty}(-1)^{J} \sum_{J_{1}+J_{2}=\jmath}\left(\begin{array}{c}
J \\
J_{1}, J_{2}
\end{array}\right)(-3)^{J_{2}} \\
& \times e^{a^{\rho}}\left(\frac{\varkappa^{\rho}-a^{\rho}}{\rho}\right)^{0.1 j_{1}+0.6 J_{2}+0.8} \\
& \times \sum_{i=0}^{\infty} \frac{\left(\varkappa^{\rho}-a^{\rho}\right)^{i}}{\Gamma\left(0.1 J_{1}+0.6 J_{2}+0.8+i+1\right)} .
\end{aligned}
$$

The next theorem proves the uniqueness result of Eq. (1.1).

Theorem 3.5 Suppose that $\mathbb{F}:[a, b] \times \mathbb{R} \rightarrow \mathbb{R}$ is a continuous function, and there exists a constant $C$ such that

$$
\left|\mathbb{F}\left(\varkappa, \varpi_{1}\right)-\mathbb{F}\left(\varkappa, \varpi_{2}\right)\right| \leq C\left|\varpi_{1}-\varpi_{2}\right|, \quad \varkappa \in[a, b], \varpi_{1}, \varpi_{2} \in \mathbb{R} .
$$

In addition, if

$$
C \kappa E_{\left(\alpha_{n}-\alpha_{n-1}, \ldots, \alpha_{n}-\alpha_{0}, \alpha_{n}+1\right)}\left(\left|a_{n-1}\right|\left(\frac{b^{\rho}-a^{\rho}}{\rho}\right)^{\left(\alpha_{n}-\alpha_{n-1}\right)}, \ldots,\left|a_{0}\right|\left(\frac{b^{\rho}-a^{\rho}}{\rho}\right)^{\left(\alpha_{n}-\alpha_{0}\right)}\right)
$$

$<1$,

then problem FIDE (1.1) has a unique solution on $A C_{0}[a, b]$.

Proof Define the operator $\mathfrak{L}$ on $A C_{0}[a, b]$ by

$$
\begin{aligned}
& \mathfrak{L}(\varpi)=\sum_{J=0}^{\infty}(-1)^{J} \sum_{J_{1}+\cdots+J_{n}=J}\left(\begin{array}{c}
J \\
J_{1}, J_{2}, \ldots, J_{n}
\end{array}\right) a_{n-1}^{J_{1}} \cdots a_{0}^{J_{n}} \\
& \times{ }^{\rho} I_{a}^{J_{1}}\left(\alpha_{n}-\alpha_{n-1}\right)+\cdots+J_{n}\left(\alpha_{n}-\alpha_{0}\right)+\alpha_{n} \int_{a}^{\varkappa} \mathbb{F}\left(\tau, \varpi^{\prime}(\tau)\right) d \tau .
\end{aligned}
$$

Let $\varpi \in A C_{0}[a, b]$. Then

$$
\int_{a}^{\varkappa} \mathbb{F}\left(\tau, \varpi^{\prime}(\tau)\right) d \tau \in A C_{0}[a, b]
$$

as $\varpi^{\prime}(\tau) \in L(a, b)$ and $\mathbb{F}\left(\tau, \varpi^{\prime}(\tau)\right) \in L(a, b)$. Obviously,

$$
\begin{aligned}
\left\|\int_{a}^{\varkappa} \mathbb{F}\left(\tau, \varpi^{\prime}(\tau)\right) d \tau\right\|_{0} & =\int_{a}^{b}\left|\mathbb{F}\left(\varkappa, \varpi^{\prime}(\varkappa)\right)\right| d \varkappa \\
& =\int_{a}^{b}\left|\mathbb{F}\left(\varkappa, \varpi^{\prime}(\varkappa)\right)-\mathbb{F}(\varkappa, 0)+\mathbb{F}(\varkappa, 0)\right| d \varkappa \\
& \leq \int_{a}^{b}\left|\mathbb{F}\left(\varkappa, \varpi^{\prime}(\varkappa)\right)-\mathbb{F}(\varkappa, 0)\right| d \varkappa+\int_{a}^{b}|\mathbb{F}(\varkappa, 0)| d \varkappa \\
& \leq C \int_{a}^{b}\left|\varpi^{\prime}(\varkappa)\right| d \varkappa+\int_{a}^{b}|\mathbb{F}(\varkappa, 0)| d \varkappa<\infty .
\end{aligned}
$$


Inequality (3.4) shows that

$$
\|\mathfrak{L}(\varpi)\|_{0}<\infty \quad \text { and } \quad \mathfrak{L}(\varpi)(a)=0
$$

Moreover, $\mathfrak{L}(\varpi)$ is absolutely continuous on $[a, b]$ by Theorem 3.1. Consequently, $\mathfrak{L}$ : $A C_{0}[a, b] \rightarrow A C_{0}[a, b]$. It remains to show that $\mathfrak{L}$ is a contraction. To this end, let $\varpi, \varpi^{*} \in$ $A C_{0}[a, b]$. Then

$$
\begin{aligned}
\left\|\mathfrak{L}(\varpi)-\mathfrak{L}\left(\varpi^{*}\right)\right\|_{0} \leq & \kappa E_{\left(\alpha_{n}-\alpha_{n-1}, \ldots, \alpha_{n}-\alpha_{0}, \alpha_{n}+1\right)}\left(\left|a_{n-1}\right|\left(\frac{b^{\rho}-a^{\rho}}{\rho}\right)^{\alpha_{n}-\alpha_{n-1}},\right. \\
& \left.\ldots,\left|a_{0}\right|\left(\frac{b^{\rho}-a^{\rho}}{\rho}\right)^{\alpha_{n}-\alpha_{0}}\right) \\
& \times\left\|\int_{a}^{\varkappa} \mathbb{F}\left(\tau, \varpi^{\prime}(\tau)\right) d \tau-\int_{a}^{\varkappa} \mathbb{F}\left(\tau, \varpi^{* \prime}(\tau)\right) d \tau\right\|_{0} .
\end{aligned}
$$

Since

$$
\begin{aligned}
\left\|\int_{a}^{\varkappa} \mathbb{F}\left(\tau, \varpi^{\prime}(\tau)\right) d \tau-\int_{a}^{\varkappa} \mathbb{F}\left(\tau, \varpi^{* \prime}(\tau)\right) d \tau\right\|_{0} & =\int_{a}^{b}\left|\mathbb{F}\left(\varkappa, \varpi^{\prime}(\varkappa)\right)-\mathbb{F}\left(\varkappa, \varpi^{* \prime}(\varkappa)\right)\right| d \varkappa \\
& \leq C \int_{a}^{b}\left|\varpi^{\prime}-\varpi^{* \prime}\right| d \varkappa \\
& =C\left\|\varpi-\varpi^{*}\right\|_{0},
\end{aligned}
$$

we get

$$
\begin{gathered}
\left\|\mathfrak{L}(\varpi)-\mathfrak{L}\left(\varpi^{*}\right)\right\|_{0} \leq \\
\ldots \kappa E_{\left(\alpha_{n}-\alpha_{n-1}, \ldots, \alpha_{n}-\alpha_{0}, \alpha_{n}+1\right)}\left(\left|a_{n-1}\right|\left(\frac{b^{\rho}-a^{\rho}}{\rho}\right)^{\alpha_{n}-\alpha_{n-1}},\right. \\
\left.\ldots,\left|a_{0}\right|\left(\frac{b^{\rho}-a^{\rho}}{\rho}\right)^{\alpha_{n}-\alpha_{0}}\right)\left\|\varpi-\varpi^{*}\right\|_{0}
\end{gathered}
$$

Inequality (3.5) shows that $\mathfrak{L}$ is contractive. The proof is done.

\section{Ulam-Hyers stability (UHS)}

Here, we develop and give some recent results on the UHS and generalized UHS of system (1.1). For $\epsilon>0$ and $\varpi_{1} \in A C_{0}[a, b]$, we consider the following inequality:

$$
\begin{gathered}
\mid{ }^{C} D_{a}^{\rho, \alpha_{n}} \varpi_{1}(\varkappa)+a_{n-1}{ }^{C} D_{a}^{\rho, \alpha_{n-1}} \varpi_{1}(\varkappa)+\cdots+a_{0}{ }^{C} D_{a}^{\rho, \alpha_{0}} \varpi_{1}(\varkappa) \\
-\int_{a}^{\varkappa} \mathbb{F}\left(\tau, \varpi^{\prime}(\tau)\right) d \tau \mid \leq \epsilon, \quad \varkappa \in[a, b] .
\end{gathered}
$$

Remark 4.1 Let $\epsilon>0$. The function $\varpi_{1} \in A C_{0}[a, b]$ satisfies (4.1) if and only if there exists a small perturbation $\zeta(\varkappa) \in A C_{0}[a, b]$ with $\zeta(0)=0$ such that

(i) $\|\zeta\|_{0}=\int_{a}^{\varkappa}\left|\zeta^{\prime}(\tau)\right| d \tau \leq \epsilon$, for $\varkappa \in[a, b]$, 
Kassim et al. Advances in Difference Equations

(2021) 2021:375

Page 13 of 18

(ii) For $\varkappa \in[a, b]$,

$$
\left\{\begin{array}{l}
{ }^{C} D_{a}^{\rho, \alpha_{n}} \varpi_{1}(\varkappa)+a_{n-1}{ }^{C} D_{a}^{\rho, \alpha_{n-1}} \varpi_{1}(\varkappa)+\cdots+a_{0}{ }^{C} D_{a}^{\rho, \alpha_{0}} \varpi_{1}(\varkappa) \\
=\int_{a}^{\varkappa} \mathbb{F}\left(\tau, \varpi_{1}^{\prime}(\tau)\right) d \tau+\int_{a}^{\varkappa}\left|\zeta^{\prime}(\tau)\right| d \tau .
\end{array}\right.
$$

Lemma 4.2 The solution of perturbed problem (4.2) with the condition $\varpi_{1}(a)=0$ satisfies the following inequality:

$$
\begin{gathered}
\left\|\varpi_{1}-Z_{\mathbb{F}}\right\|_{0} \leq \kappa E_{\left(\alpha_{n}-\alpha_{n-1}, \ldots, \alpha_{n}-\alpha_{0}, \alpha_{n}+1\right)}\left(\left|a_{n-1}\right|\left(\frac{b^{\rho}-a^{\rho}}{\rho}\right)^{\alpha_{n}-\alpha_{n-1}}\right. \\
\left.\ldots,\left|a_{0}\right|\left(\frac{b^{\rho}-a^{\rho}}{\rho}\right)^{\alpha_{n}-\alpha_{0}}\right) \epsilon
\end{gathered}
$$

where

$$
\begin{aligned}
Z_{\mathbb{F}}(\varkappa):= & \sum_{J=0}^{\infty}(-1)^{J} \sum_{J_{1}+\cdots+J_{n}=J}\left(\begin{array}{c}
J \\
J_{1}, J_{2}, \ldots, J_{n}
\end{array}\right) \\
& \times a_{n-1}^{j 1} \cdots a_{0}^{J_{n} \rho} I_{a}^{J_{1}\left(\alpha_{n}-\alpha_{n-1}\right)+\cdots+J_{n}\left(\alpha_{n}-\alpha_{0}\right)+\alpha_{n}} \int_{a}^{\varkappa} \mathbb{F}\left(\tau, \varpi_{1}^{\prime}(\tau)\right) d \tau
\end{aligned}
$$

and $\kappa$ is defined by (3.3).

Proof By Theorem 3.1, the solution of perturbed problem (4.2) is given by

$$
\begin{aligned}
& \varpi_{1}(\varkappa)=\sum_{J=0}^{\infty}(-1)^{J} \sum_{J_{1}+\cdots+J_{n}=J}\left(\begin{array}{c}
J \\
J_{1}, J_{2}, \ldots, J_{n}
\end{array}\right) a_{n-1}^{J_{1}} \cdots a_{0}^{J_{n}} \\
& \times{ }^{\rho} I_{a}^{j 1}\left(\alpha_{n}-\alpha_{n-1}\right)+\cdots+J_{n}\left(\alpha_{n}-\alpha_{0}\right)+\alpha_{n}\left[\int_{a}^{\varkappa} \mathbb{F}\left(\tau, \varpi_{1}^{\prime}(\tau)\right) d \tau+\int_{a}^{\varkappa}\left|\zeta^{\prime}(\tau)\right| d \tau\right] .
\end{aligned}
$$

From Eq. (4.3), Remark 4.1, and Eq. (3.4), we get

$$
\begin{aligned}
\left\|\varpi_{1}-Z_{\mathbb{F}}\right\|_{0} \leq & \kappa E_{\left(\alpha_{n}-\alpha_{n-1}, \ldots, \alpha_{n}-\alpha_{0}, \alpha_{n}+1\right)}\left(\left|a_{n-1}\right|\left(\frac{b^{\rho}-a^{\rho}}{\rho}\right)^{\alpha_{n}-\alpha_{n-1}},\right. \\
& \left.\ldots,\left|a_{0}\right|\left(\frac{b^{\rho}-a^{\rho}}{\rho}\right)^{\alpha_{n}-\alpha_{0}}\right) \\
& \times\left\|\int_{a}^{\varkappa} \mathbb{F}\left(\tau, \varpi_{1}^{\prime}(\tau)\right) d \tau+\int_{a}^{\varkappa}\left|\zeta^{\prime}(\tau)\right| d \tau-\int_{a}^{\varkappa} \mathbb{F}\left(\tau, \varpi_{1}^{\prime}(\tau)\right) d \tau\right\|_{0} \\
\leq & \kappa E_{\left(\alpha_{n}-\alpha_{n-1}, \ldots, \alpha_{n}-\alpha_{0}, \alpha_{n}+1\right)}\left(\left|a_{n-1}\right|\left(\frac{b^{\rho}-a^{\rho}}{\rho}\right)^{\alpha_{n}-\alpha_{n-1}},\right. \\
& \left.\ldots,\left|a_{0}\right|\left(\frac{b^{\rho}-a^{\rho}}{\rho}\right)^{\alpha_{n}-\alpha_{0}}\right)\|\zeta\|_{0} \\
\leq & \kappa E_{\left(\alpha_{n}-\alpha_{n-1}, \ldots, \alpha_{n}-\alpha_{0}, \alpha_{n}+1\right)}\left(\left|a_{n-1}\right|\left(\frac{b^{\rho}-a^{\rho}}{\rho}\right)^{\alpha_{n}-\alpha_{n-1}}\right. \\
& \left.\ldots,\left|a_{0}\right|\left(\frac{b^{\rho}-a^{\rho}}{\rho}\right)^{\alpha_{n}-\alpha_{0}}\right) \epsilon .
\end{aligned}
$$


Theorem 4.3 (UHS) Assume that the assumptions of Theorem 3.5 and (4.1) hold. Then problem (1.1) is UH stable.

Proof Let $\epsilon>0$ and $\varpi_{1} \in A C_{0}[a, b]$ satisfy (4.1), and let $\varpi \in A C_{0}[a, b]$ be a unique solution of

$$
\left\{\begin{array}{l}
{ }^{C} D_{a}^{\rho, \alpha_{n}} \varpi(\varkappa)+a_{n-1}{ }^{C} D_{a}^{\rho, \alpha_{n-1}} \varpi(\varkappa)+\cdots+a_{0}{ }^{C} D_{a}^{\rho, \alpha_{0}} \varpi(\varkappa)=\int_{a}^{\varkappa} \mathbb{F}\left(\tau, \varpi^{\prime}(\tau)\right) d \tau, \\
\varpi(a)=\varpi_{1}(a)=0,
\end{array}\right.
$$

that is,

$$
\begin{aligned}
\varpi(\varkappa)= & \varpi(a)+\sum_{j=0}^{\infty}(-1)^{\jmath} \sum_{J_{1}+\cdots+J_{n}=J}\left(\begin{array}{c}
J \\
J_{1}, J_{2}, \ldots, J_{n}
\end{array}\right) \\
& \times a_{n-1}^{J_{1}} \cdots a_{0}^{J n \rho} I_{a}^{J_{1}\left(\alpha_{n}-\alpha_{n-1}\right)+\cdots+J_{n}\left(\alpha_{n}-\alpha_{0}\right)+\alpha_{n}}\left[\int_{a}^{\varkappa} \mathbb{F}\left(\tau, \varpi^{\prime}(\tau)\right) d \tau\right] .
\end{aligned}
$$

Since $\varpi(a)=\varpi_{1}(a)=0$, we get

$$
\begin{aligned}
\varpi(\varkappa)= & \sum_{j=0}^{\infty}(-1)^{j} \sum_{J_{1}+\cdots+J_{n}=J}\left(\begin{array}{c}
J \\
J_{1}, J_{2}, \ldots, J_{n}
\end{array}\right) \\
& \times a_{n-1}^{J_{1}} \cdots a_{0}^{J_{n} \rho} I_{a}^{J_{1}\left(\alpha_{n}-\alpha_{n-1}\right)+\cdots+J_{n}\left(\alpha_{n}-\alpha_{0}\right)+\alpha_{n}}\left[\int_{a}^{\varkappa} \mathbb{F}\left(\tau, \varpi^{\prime}(\tau)\right) d \tau\right] .
\end{aligned}
$$

By virtue of Lemma 4.2 and Eq. (3.4), we have

$$
\begin{aligned}
\left\|\varpi_{1}-\varpi\right\|_{0} \leq & \left\|\varpi_{1}-Z_{\mathbb{F}}\right\|_{0}+\left\|Z_{\mathbb{F}}-\varpi\right\|_{0} \\
\leq & \kappa E_{\left(\alpha_{n}-\alpha_{n-1}, \ldots, \alpha_{n}-\alpha_{0}, \alpha_{n}+1\right)}\left(\left|a_{n-1}\right|\left(\frac{b^{\rho}-a^{\rho}}{\rho}\right)^{\alpha_{n}-\alpha_{n-1}},\right. \\
& \left.\ldots,\left|a_{0}\right|\left(\frac{b^{\rho}-a^{\rho}}{\rho}\right)^{\alpha_{n}-\alpha_{0}}\right) \epsilon \\
& +\kappa E_{\left(\alpha_{n}-\alpha_{n-1}, \ldots, \alpha_{n}-\alpha_{0}, \alpha_{n}+1\right)}\left(\left|a_{n-1}\right|\left(\frac{b^{\rho}-a^{\rho}}{\rho}\right)^{\alpha_{n}-\alpha_{n-1}},\right. \\
& \left.\ldots,\left|a_{0}\right|\left(\frac{b^{\rho}-a^{\rho}}{\rho}\right)^{\alpha_{n}-\alpha_{0}}\right) \\
& \times\left\|\int_{a}^{\varkappa} \mathbb{F}\left(\tau, \varpi_{1}^{\prime}(\tau)\right) d \tau-\int_{a}^{\varkappa} \mathbb{F}\left(\tau, \varpi^{\prime}(\tau)\right) d \tau\right\|_{0}
\end{aligned}
$$

Using the assumptions of Theorem 3.5, we obtain

$$
\left\|\int_{a}^{\varkappa} \mathbb{F}\left(\tau, \varpi_{1}^{\prime}(\tau)\right) d \tau-\int_{a}^{\varkappa} \mathbb{F}\left(\tau, \varpi^{\prime}(\tau)\right) d \tau\right\|_{0} \leq C\left\|\varpi_{1}-\varpi\right\|_{0} .
$$

Consequently,

$$
\left\|\varpi_{1}-\varpi\right\|_{0} \leq \kappa E_{\left(\alpha_{n}-\alpha_{n-1}, \ldots, \alpha_{n}-\alpha_{0}, \alpha_{n}+1\right)}\left(\left|a_{n-1}\right|\left(\frac{b^{\rho}-a^{\rho}}{\rho}\right)^{\alpha_{n}-\alpha_{n-1}},\right.
$$




$$
\begin{aligned}
& \left.\ldots,\left|a_{0}\right|\left(\frac{b^{\rho}-a^{\rho}}{\rho}\right)^{\alpha_{n}-\alpha_{0}}\right) \epsilon \\
& +\kappa E_{\left(\alpha_{n}-\alpha_{n-1}, \ldots, \alpha_{n}-\alpha_{0}, \alpha_{n}+1\right)}\left(\left|a_{n-1}\right|\left(\frac{b^{\rho}-a^{\rho}}{\rho}\right)^{\alpha_{n}-\alpha_{n-1}},\right. \\
& \left.\ldots,\left|a_{0}\right|\left(\frac{b^{\rho}-a^{\rho}}{\rho}\right)^{\alpha_{n}-\alpha_{0}}\right) C\left\|\varpi_{1}-\varpi\right\|_{0} .
\end{aligned}
$$

By dint of inequality (3.5), we conclude that

$$
\left\|\varpi_{1}-\varpi\right\|_{0} \leq C_{\mathbb{F}} \epsilon,
$$

where $C_{\mathbb{F}}:=\frac{\mathfrak{R}}{1-\mathfrak{R C}}$ and

$$
\mathfrak{R}:=\kappa E_{\left(\alpha_{n}-\alpha_{n-1}, \ldots, \alpha_{n}-\alpha_{0}, \alpha_{n}+1\right)}\left(\left|a_{n-1}\right|\left(\frac{b^{\rho}-a^{\rho}}{\rho}\right)^{\alpha_{n}-\alpha_{n-1}}, \ldots,\left|a_{0}\right|\left(\frac{b^{\rho}-a^{\rho}}{\rho}\right)^{\alpha_{n}-\alpha_{0}}\right) .
$$

Conclusion 4.4 Under assumptions of Theorem 4.3, if we set $\Phi(\epsilon)=C_{\mathbb{F}} \epsilon$ such that $\Phi(0)=$ 0 , then problem (1.1) is generalized Ulam-Hyers stable.

Example 4.5 Let $a=1$ and $b=\sqrt[\rho]{1+\rho}$. Then there is a unique solution for the following Katugampola-type FIDE:

$$
\begin{gathered}
{ }^{C} D_{a}^{\rho, 0.9} \varpi(\varkappa)-{ }^{C} D_{a}^{\rho, 0.4} \varpi(\varkappa)+{ }^{C} D_{a}^{\rho, 0.3} \varpi(\varkappa)-{ }^{C} D_{a}^{\rho, 0.1} \varpi(\varkappa) \\
=\int_{a}^{\varkappa}\left(\frac{e^{\tau^{2}}}{C\left(3+e^{\tau^{2}}\right)} \sin \varpi^{\prime}(\tau)+e^{\cos \tau}+\ln (1+\sqrt{\tau})\right) d \tau,
\end{gathered}
$$

where the constant $C$ is to be determined.

Clearly, the function

$$
\mathbb{F}(\varkappa, z)=\frac{e^{\varkappa^{2}}}{C\left(3+e^{\varkappa^{2}}\right)} \sin z+e^{\cos \varkappa}+\ln (1+\sqrt{\varkappa})
$$

is a continuous function from $[1, \sqrt[\rho]{1+\rho}] \times \mathbb{R}$ to $\mathbb{R}$ and satisfies

$$
\begin{aligned}
\left|\mathbb{F}\left(\varkappa, z_{1}\right)-\mathbb{F}\left(\varkappa, z_{2}\right)\right| & =\left|\frac{e^{\varkappa^{2}}}{C\left(3+e^{\varkappa^{2}}\right)} \sin z_{1}-\frac{e^{\varkappa^{2}}}{C\left(3+e^{\varkappa^{2}}\right)} \sin z_{1}\right| \\
& \leq \frac{e^{\varkappa^{2}}}{C\left(3+e^{\varkappa^{2}}\right)}\left|\sin z_{1}-\sin z_{1}\right| \\
& \leq \frac{e^{\varkappa^{2}}}{C\left(3+e^{\varkappa^{2}}\right)}\left|z_{1}-z_{1}\right| \leq \frac{1}{C}\left|z_{1}-z_{1}\right| .
\end{aligned}
$$

Obviously $\frac{b^{\rho}-a^{\rho}}{\rho}=1$ and

$$
\begin{aligned}
& \sum_{J=0}^{\infty} \sum_{J 1+J_{2}+J_{3}=J}\left(\begin{array}{c}
J \\
J_{1}, J_{2}, J_{3}
\end{array}\right) \\
& \quad \times\left(|-1|\left(\frac{b^{\rho}-a^{\rho}}{\rho}\right)^{0.5}\right)^{J 1}\left(|1|\left(\frac{b^{\rho}-a^{\rho}}{\rho}\right)^{0.6}\right)^{J 2}\left(|-1|\left(\frac{b^{\rho}-a^{\rho}}{\rho}\right)^{0.8}\right)^{J 3}
\end{aligned}
$$




$$
\begin{aligned}
& \times \frac{1}{\Gamma\left(0.5 J_{1}+0.6 J_{2}+0.8 J_{3}+1.9\right)} \\
= & \sum_{J=0}^{\infty} \sum_{J_{1}+J_{2}+J_{3}=J}\left(\begin{array}{c}
J \\
J_{1}, J_{2}, J_{3}
\end{array}\right) \frac{1}{\Gamma\left(0.5 J_{1}+0.6 J_{2}+0.8 J_{3}+1.9\right)} \\
= & E_{(0.5,0.6,6,0.8,1.9)}(1,1,1) .
\end{aligned}
$$

Then we choose a positive $C$ such that

$$
C \kappa E_{(0.5,0.6,0.8,1.9)}(1,1,1)<1 \text {. }
$$

According to Theorem 3.5, Eq. (4.4) has a unique solution.

Furthermore, by Theorem 4.3, for any solution $\varpi_{1}(\varkappa) \in A C_{0}[a, b]$ of the inequality

$$
\begin{aligned}
& \mid{ }^{C} D_{a}^{\rho, 0.9} \varpi_{1}(\varkappa)-{ }^{C} D_{a}^{\rho, 0.4} \varpi_{1}(\varkappa)+{ }^{C} D_{a}^{\rho, 0.3} \varpi_{1}(\varkappa)-{ }^{C} D_{a}^{\rho, 0.1} \varpi_{1}(\varkappa) \\
& \quad-\int_{a}^{\varkappa}\left(\frac{e^{\tau^{2}}}{C\left(3+e^{\tau^{2}}\right)} \sin \varpi_{1}^{\prime}(\tau)+e^{\cos \tau}+\ln (1+\sqrt{\tau})\right) d \tau \mid \leq \epsilon, \quad \varkappa \in[a, b],
\end{aligned}
$$

there exists a unique solution $\varpi(\varkappa) \in A C_{0}[a, b]$ of Eq. (4.4) such that

$$
\left\|\varpi_{1}-\varpi\right\|_{0} \leq C_{\mathbb{F}} \epsilon
$$

where $C_{\mathbb{F}}:=\frac{\mathfrak{R}}{1-\mathfrak{R C} C}>0, \mathfrak{R}=\kappa E_{(0.5,0.6,0.8,1.9)}(1,1,1)$, and $\kappa=\frac{1}{\Gamma\left(0.5 J_{1}+0.6 J_{2}+0.8 J_{3}+1.9\right)}$. Hence Eq. (4.4) is UH stable.

Remark 4.6 The results obtained in this work will remain valid if we use generalized Riemann-Liouville-type instead of generalized Caputo-type in the proposed problem (1.1). Specifically, in problem (1.1), if we replace Caputo derivatives with RiemannLiouville derivatives, then Lemma 2.9 is valid with respect to ${ }^{R L} D_{a}^{\rho, \alpha}(\cdot)$, due to $\vartheta(a)=0$ whenever $\vartheta \in A C_{0}[a, b]$.

\section{Conclusions}

Using the Banach space $A C_{0}[a, b]$, Banach's fixed point technique, and Babenko's method, we have obtained the uniqueness of solutions for nonlinear FIDE (1.1) with generalized Caputo fractional derivatives. Moreover, we have proven various types of stability analysis of the suggested problem. Also, some pertinent examples are given to substantiate the main results. The reported results in this study extend and develop the presented study by $\mathrm{Li}[38]$.

As future work, we are thinking of extending the current results to include more generalized operators such as $\psi$-Caputo [52].

\section{Acknowledgements}

The authors T. Abdeljawad and W. Shatanawi would like to thank Prince Sultan University for the moral support to this work through the research group Nonlinear Analysis Methods in Applied Mathematics (NAMAM) group number RG-DES-2017-01-17. The authors also thank the reviewers for their valuable remarks on our work. 
Availability of data and materials

This is not applicable in our paper.

\section{Competing interests}

The authors declare that they have no competing interests.

\section{Authors' contributions}

All authors contributed this manuscript and approved the final version.

\section{Author details}

'Department of Basic Engineering Sciences, College of Engineering, Imam Abdulrahman Bin Faisal University, P.O. Box 1982, Dammam 34151, Saudi Arabia. ${ }^{2}$ Department of Mathematics and General Sciences, Prince Sultan University, Riyadh, Saudi Arabia. ${ }^{3}$ Department of Medical Research, China Medical University, Taichung 40402, Taiwan. ${ }^{4}$ Department of Computer Science and Information Engineering, Asia University, Taichung, Taiwan. ${ }^{5}$ Department of Mathematics, Hodeidah University, Al-Hudeidah, Yemen.

\section{Publisher's Note}

Springer Nature remains neutral with regard to jurisdictional claims in published maps and institutional affiliations.

Received: 8 March 2021 Accepted: 28 July 2021 Published online: 10 August 2021

\section{References}

1. Bagley, R.L., Torvik, P.J.: A theoretical basis for the application of fractional calculus to viscoelasticity. J. Rheol. 27, 201-210 (1983)

2. Bagley, R.L., Torvik, P.J.: A different approach to the analysis of viscoelastically damped structures. AIAA J. 21, 741-748 (1983)

3. Bagley, R.L., Torvik, P.J.: On the appearance of the fractional derivative in the behavior of real material. J. Appl. Mech. 51, 294-298 (1983)

4. Zhang, L., Ahmad, B., Wang, G., Agarwal, R.P.: Nonlinear fractional integro-differential equations on unbounded domains in a Banach space. J. Comput. Appl. Math. 249, 51-56 (2013)

5. Ahmad, B., Ntouyas, S.K.: On Hadamard fractional integro-differential boundary value problems. J. Appl. Math. Comput. 47(1), 119-131 (2015)

6. Benchohra, M., Ntouyas, S.K.: Nonlocal Cauchy problems for neutral functional differential and integrodifferential inclusions in Banach spaces. J. Math. Anal. Appl. 258(2), 573-590 (2001)

7. Benchohra, M., Bouriah, S., Nieto Roig, J.J.: Existence and Ulam stability for nonlinear implicit differential equations with Riemann-Liouville fractional derivative. Demonstr. Math. 52(1), 437-450 (2019)

8. Ravichandran, C., Logeswari, K., Jarad, F.: New results on existence in the framework of Atangana-Baleanu derivative for fractional integro-differential equations. Chaos Solitons Fractals 125, 194-200 (2019)

9. Aghajani, A., Jalilian, Y., Trujillo, J.J.: On the existence of solutions of fractional integro-differential equations. Fract. Calc. Appl. Anal. 15(1), 44-69 (2012)

10. Khan, A., Li, Y., Shah, K., Khan, T.S.: On coupled p-Laplacian fractional differential equations with nonlinear boundary conditions. Complexity 2017, Article ID 8197610 (2017). https://doi.org/10.1155/2017/8197610

11. Hasib, K., Wen, C., Aziz, K., Tahir, S.K., Qasem, M.A.: Hyers-Ulam stability and existence criteria for coupled fractional differential equations involving p-Laplacian operator. Adv. Differ. Equ. 2018, 455 (2018)

12. Ardjouni, A.: Asymptotic stability in Caputo-Hadamard fractional dynamic equations. Results Nonlinear Anal. 4(2), 77-86 (2021)

13. Fatima, S.B., Saïd, A., Maamar, B., Mouffak, B.: Hilfer-Hadamard fractional differential equations; existence and attractivity. Adv. Theory Nonlinear Anal. Appl. 5(1), 49-57 (2021)

14. Tuan, N.H., Baleanu, D., Thach, T.N., O'Regan, D., Can, N.H.: Final value problem for nonlinear time fractional reaction-diffusion equation with discrete data. J. Comput. Appl. Math. 376, 112883 (2020)

15. Mohamed, H.: Existence of solutions for a coupled system of Caputo-Hadamard type fractional differential equations with Hadamard fractional integral conditions. Adv. Theory Nonlinear Anal. Appl. 5(3), 316-329 (2021)

16. Redhwan, S.S., Shaikh, S.L., Abdo, M.S.: Implicit fractional differential equation with anti-periodic boundary condition involving Caputo-Katugampola type. AIMS Math. 5(4), 3714-3730 (2020)

17. Afshari, H., Kalantari, S., Karapınar, E.: Solution of fractional differential equations via coupled fixed point. Electron. J. Differ. Equ. 2015(286), 1 (2015)

18. Abdo, M.S., Abdeljawad, T., Shah, K., Jarad, F.: Study of impulsive problems under Mittag-Leffler power law. Heliyon 6(10), e05109 (2020)

19. Sevinik, A.R., Aksoy, Ü., Karapınar, E., Erhan, I.M.: On the solution of a boundary value problem associated with a fractional differential equation. Math. Methods Appl. Sci. (2020). https://doi.org/10.1002/mma.6652

20. Afshari, H., Kalantari, S., Karapınar, E.: Solution of fractional differential equations via coupled fixed point. Electron. J. Differ. Equ. 2015, 286 (2015)

21. Alqahtani, B., Aydi, H., Karapınar, E., Rakočević, V.: A solution for Volterra fractional integral equations by hybrid contractions. Mathematics 7(8), 694 (2019)

22. Karapınar, E., Fulga, A., Rashid, M., Shahid, L., Aydi, H.: Large contractions on quasi-metric spaces with an application to nonlinear fractional differential equations. Mathematics 7(5), 444 (2019)

23. Abdeljawad, T., Agarwal, R.P., Karapınar, E., Kumari, P.S.: Solutions of the nonlinear integral equation and fractional differential equation using the technique of a fixed point with a numerical experiment in extended b-metric space. Symmetry 11(5), 686 (2019)

24. Almalahi, M.A., Panchal, S.K.: Some existence and stability results for $\psi$-Hilfer fractional implicit differential equation with periodic conditions. J. Math. Anal. Model. 1(1), 1-19 (2020) 
25. Abdo, M.S., Abdeljawad, T., Ali, S.M., Shah, K.: On fractional boundary value problems involving fractional derivatives with Mittag-Leffler kernel and nonlinear integral conditions. Adv. Differ. Equ. 2021, 37 (2021). https://doi.org/10.1186/s13662-020-03196-6

26. Abdo, M.S., Panchal, S.K., Wahash, H.A.: Ulam-Hyers-Mittag-Leffler stability for a $\psi$-Hilfer problem with fractional order and infinite delay. Results Appl. Math. 7, 100115 (2020). https://doi.org/10.1016/j.rinam.2020.100115

27. Abdo, M.S., Thabet, S.T., Ahmad, B.: The existence and Ulam-Hyers stability results for $\psi$-Hilfer fractional integrodifferential equations. J. Pseudo-Differ. Oper. Appl. 11(4), 1757-1780 (2020)

28. Abdo, M.S., Panchal, S.K.: Some new uniqueness results of solutions to nonlinear fractional integro-differential equations. Ann. Pure Appl. Math. 16(2), 345-352 (2018)

29. Abdo, M.S.: Further results on the existence of solutions for generalized fractional quadratic functional integral equations. J. Math. Anal. Model. 1(1), 33-46 (2020)

30. Zidane, B., Choukri, D., Mouffak, B.: $\psi$-Caputo fractional differential equations with multi-point boundary conditions by topological degree theory. Results Nonlinear Anal. 3(4), 166-178 (2020)

31. Afshari, H., Karapınar, E.: A discussion on the existence of positive solutions of the boundary value problems via $\psi$-Hilfer fractional derivative on b-metric spaces. Adv. Differ. Equ. 2020, 616 (2020)

32. Abbas, M.I.: On the nonlinear sequential $\psi$-Hilfer fractional differential equations. Int. J. Math. Anal. 14, 77-90 (2020)

33. Kassim, M.D., Furati, K.M., Tatar, N.E.: On a differential equation involving Hilfer-Hadamard fractional derivative. Abstr. Appl. Anal. 2012, 391062 (2012)

34. Kassim, M.D., Tatar, N.E.: Well-posedness and stability for a differential problem with Hilfer-Hadamard fractional derivative. Abstr. Appl. Anal. 2013, 605029 (2013)

35. Kassim, M.D., Furati, K.M., Tatar, N.E.: Asymptotic behavior of solutions to nonlinear fractional differential equations. Math. Model. Anal. 21(5), 610-629 (2016)

36. Kassim, M.D., Furati, K.M., Tatar, N.E.: Asymptotic behavior of solutions to nonlinear initial-value fractional differential problems. Electron. J. Differ. Equ. 291, 1 (2016)

37. Kassim, M.D., Tatar, N.E.: Stability of logarithmic type for a Hadamard fractional differential problem. J. Pseudo-Differ. Oper. Appl. 11, 447-466 (2020)

38. Li, C.: Uniqueness of the Hadamard-type integral equations. Adv. Differ. Equ. 2021(1), 1 (2021)

39. Li, C.: On the nonlinear Hadamard-type integro-differential equation. Fixed Point Theory Algorithms Sci. Eng. 2021(1), $1-15(2021)$

40. Cao Labora, D., Rodríguez López, R., Nieto Roig, J.J.: Is it possible to construct a fractional derivative such that the index law holds? Prog. Fract. Differ. Appl. 4(1), 1-3 (2018)

41. Katugampola, U.: A new approach to a generalized fractional integral. Appl. Math. Comput. 218, 860-865 (2011)

42. Katugampola, U.N.: A new approach to generalized fractional derivatives. Bull. Math. Anal. Appl. 6(4), 1-15 (2014)

43. Almeida, R.: A Gronwall inequality for a general Caputo fractional operator. Math. Inequal. Appl. 20(4), 1089-1105 (2017)

44. Almeida, R., Malinowska, A.B., Odzijewicz, T.: Fractional differential equations with dependence on the Caputo-Katugampola derivative. J. Comput. Nonlinear Dyn. 11, 061017 (2016)

45. Babenkos, Y.I.: Heat and Mass Transfer. Khimiya, Leningrad (1986) (in Russian)

46. Kilbas, A.A., Srivastava, H.M., Trujillo, J.J.: Theory and Applications of Fractional Differential Equations. North-Holland Mathematics Studies, vol. 204. Elsevier, Amsterdam (2006)

47. Kataria, K.K.: A probabilistic proof of the multinomial theorem. Am. Math. Mon. 123(1), 94-96 (2016)

48. Podlubny, I.: Fractional Differential Equations. Academic Press, New York (1999)

49. Samko, S.G., Kilbas, A.A., Marichev, O.I.: Fractional Integrals and Derivatives: Theory and Applications. Gordon \& Breach, New York (1993)

50. Gradshteyn, I.S., Ryzhik, I.M.: Tables of Integrals, Series, and Products. Academic Press, New York (1980)

51. Oliveira, D.S., de Oliveira, E.C.: Hilfer-Katugampola fractional derivative. Comput. Appl. Math. 37, 3672-3690 (2018)

52. Almeida, R.: A Caputo fractional derivative of a function with respect to another function. Commun. Nonlinear Sci. Numer. Simul. 44, 460-481 (2017)

\section{Submit your manuscript to a SpringerOpen ${ }^{\odot}$ journal and benefit from:}

- Convenient online submission

- Rigorous peer review

- Open access: articles freely available online

- High visibility within the field

- Retaining the copyright to your article

Submit your next manuscript at $>$ springeropen.com 